\title{
Efficient Resource Consumption by Dynamic Clustering and Optimized Routes in Wireless Sensor Networks
}

\author{
Farzad Kiani \\ Computer Engineering Dept., Engineering and Natural Sciences Faculty, \\ Istanbul Sabahattin Zaim University, \\ Istanbul, Turkey
}

\begin{abstract}
The energy issue is an important parameter in the wireless sensor networks and should be managed in the different applications. We propose a new routing algorithm that it is energy efficient and uses different approaches as dynamic clustering, spanning tree, self-configurable routing and controls energy consuming by data-driven and power management schemas. It has two main phases. The first is consisting of the steady cluster, cluster head election and creation-spanning tree in each cluster and the second phase is data transmission. The proposed protocol is compared with four other protocols in network lifetime, network balance, and average packet delay and packet delivery. Simulation results show the proposed protocol performance in the network lifetime is about 6 per cent higher than Improved-LEACH, 21.5 per cent higher than EESR and 5.8 per cent higher than DHCO. Its improvement in packet delivery parameter is about 3.5 per cent higher than Improved-LEACH, 6.5 per cent higher than EESR and 3 per cent higher than DHCO. In addition, the performance or in packet delay is about 17 per cent higher than EESR and 6 per cent higher than DHCO but Improved-LEACH protocol has a good performance than our protocol about 4 per cent.
\end{abstract}

Keywords-Energy efficiency; data-driven; spanning tree; sleep/wake up mode; power management

\section{INTRODUCTION}

The wireless sensor network (WSN) is introduced in mid20th century [1]. They are one of the distributed systems, subsystems that are in the pervasive systems category. These networks have many applications in medicine, military, smart systems, etc. [2]. So they can be divided to wireless body sensor networks (WBSN), wireless underground sensor networks (WUSN), wireless multimedia sensor networks (WMSN) and such. They are different from the other wireless networks such as Mobile Ad-hoc Network [2]. These networks are combined of large number of mini-size sensor nodes and a few Base Stations (BS) or sink. The nodes have low battery and limited memory. The need of them was felt in many applications and began to spread gradually. Beside the sensor nodes, WSN has one or some of the BS or sink. For example, they can be a computer server. In the network environment, sensor nodes sense phenomenon then collect and process data and send to BS/sink in the end. One of the reasons of development and progression of the WSNs is using the inexpensive and affordable sensor nodes [3]. Therefore, WSNs are used in many applications such as civil, medical, military, governmental and probability-based applications as volcano [4]. At the beginning, most researchers had focused on bandwidth and Quality of Service (QoS) factors but then energy was considered due to some of the limitations of the networks as battery and memory. Despite the researchers use different techniques to different applications, limitations of WSNs are fix and without change in any application [5]. Hence, one of the most important aims in the WSN is to save energy. The others factors can be different such as QoS and bandwidth so they are the second plan in the network design [1]. There are two kinds of energy consumption between sensor nodes. The first is energy consumption in communications and the second is consumption in computations. The communications consume more energy. Therefore, minimizing communication costs is an important issue. Researchers propose different approaches for this goal such as energy efficiency by routing techniques, data aggregation, duty-cycle techniques, and topology control and medium-access decision.

WSNs are consisting of large number of sensor nodes which the nodes sense data. The data is different due to variant applications and environments [6]. These nodes process and store self-data to memory part after convert sensed physical phenomena to digital signals. Then they send them to the $\mathrm{BS} /$ sink via direct or with the aid of other sensor nodes. The scenario is seems perfect but it is not easy according to small size and limited battery of nodes. The nodes are failure prone in real applications. Therefore, management and design of networks is different from other networks. In this mode, lifetime of network will deplete as soon as before correct and complete tasks. It seems that the problem is solved by charge of sensor nodes but charging is difficult or impractical according to concept of WSNs and their applications such as spy network in enemy environment and earthquake or fire prevent networks. Also, energy issue and energy efficiency is very important.

The major goal of some methods is reach to energy efficiency. These methods are based on different approaches such as energy-aware routing protocols or energy efficiency MAC protocols, aggregation of data, sleep/wake up nodes and topology control methods [7]. It should be noted that output parameters implementation of applications are trade off one another. For example, focus on energy parameter can be cause increasing latency or decreasing system reliability [8]. 
Therefore, always relative balancing should be between parameters. Due to the above reasons, the paper concerns with the energy issue with respect to balancing reasons. The paper is based on increasing lifetime of network by energy-efficiency routing algorithms and energy conservations realize by topology control, data aggregation and sleep/weak up of nodes. The sleep/wake up technique is set of power management schema in energy conservation issues [9]. Fig. 1 shows a view of states node and energy consumption model.

The network architecture has big role in the energy efficiency of these networks. In general, it is based on three models, which are star, mesh and hybrid as shown in Fig. 2. In the star model, a single BS/sink can transmit/receive a message packet to remote sensor nodes. The nodes cannot send the message packets to each other. Home control systems are an example for the model. Their advantages are simplicity, ability to hold down the energy consumption and communications delays between the remote node and the BS/sink. On the other hand, BS/sink must be within all nodes' radio transmission range. In addition, management of the structure has depending to a single sensor node. The last two cases are disadvantages of star structure [11]. In the mesh structure, the sensor nodes can communicate together when they are within radio range of each other. This case realizes multi-hop communication between nodes. Indeed a node can send data to any node (inside self-RF or outside) by intermediate nodes. Scalability and reliability is advantage of the model. If a node is failure then a remote node still can communicate to any other node in its range, which in turn, can forward the message to the suitable place. Energy consumption in multi-hopping system is high generally. Therefore, energy issue is a problem and disadvantage of the model. Moreover, the number of hops and packet delay time increases [11]. Hybrid model is between the star and the mesh structures that provide a robust and selfaround communications network. In this model, the sensor nodes with minimum energy are not send message to other nodes and allow to them to saving energy [11].

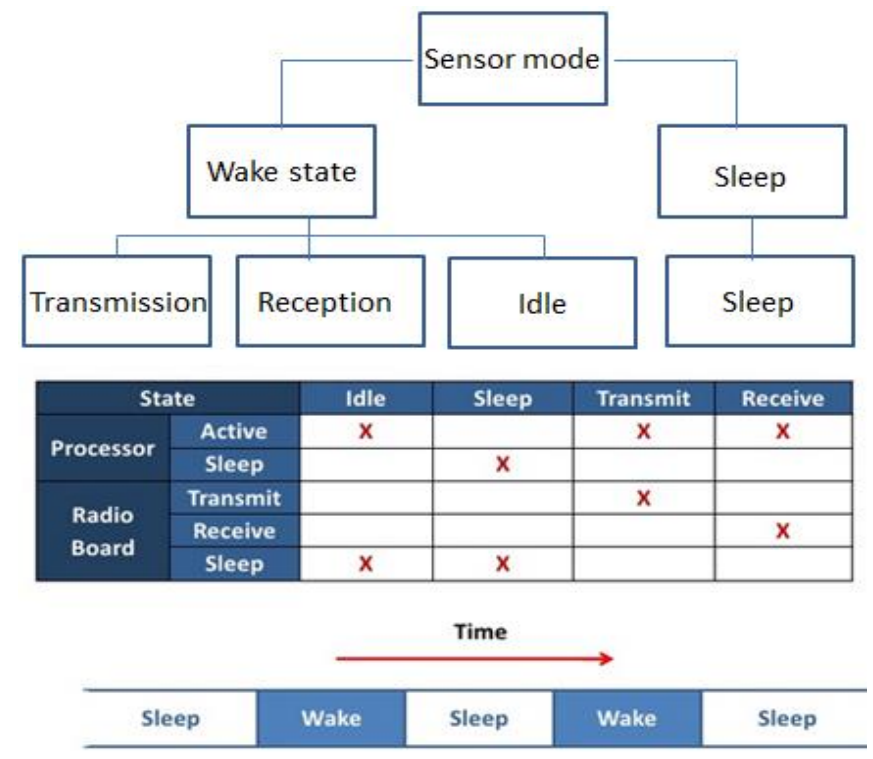

Fig. 1. A view of states node and power consumption [10].

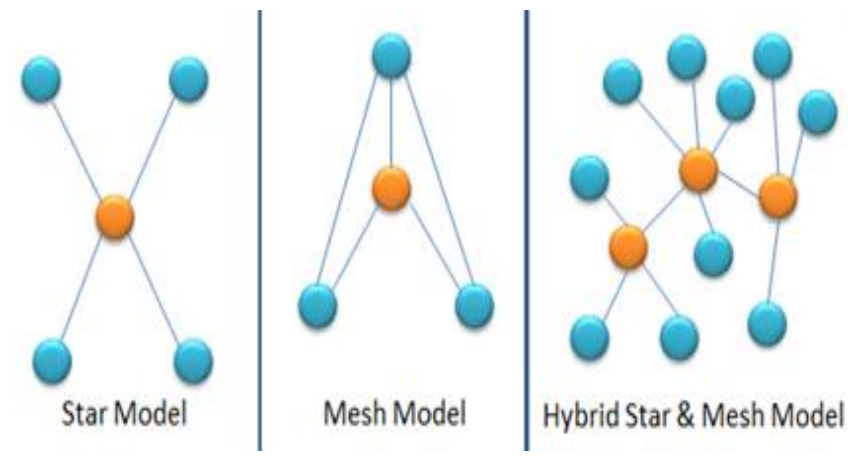

Fig. 2. Structure of a WSN [11].

In Section 2 is discussed the related works about energy efficiency in wireless sensor networks. In Section 3 is introduced a new protocol in order to increasing network lifetime by a dynamic structure that is based on clustering and spanning tree. The results of simulation and evaluation are explained in Section 4. Last section presents the conclusion and future works of the paper.

\section{RELATED WORKS}

The WSN applications domain are increasing day by day especially in Internet of Things (IoT) based applications [12]. In general, the efficient resource management is essentially issue in these networks. The energy efficiency is converted to popular issue and many of researchers focus on it. Energy efficiency is debatable in all layers of protocol stack. For example, in [13] collision, packet overhead, latency, overhearing and idle listening are discussed and focus on their management to reach energy efficiency. Collisions must be control because they cause unnecessary receive costs at the destination sensor node and cause undue send costs at the source sensor node. The collisions can are managed in the design phase by TDMA and so on protocols and in after the design by avoidance protocols.

In overhearing case is inevitable item in WSNs because the networks have many sensor nodes in an environment and broadcast data transferring is a widespread method. Therefore, possibility of overhearing the nodes within the network is perfectly normal but it is causing increasing energy consumption in the network. Hence, it must be control by some of the approaches as management of nodes density [13], [14]. Latency is gained by delays of transmitted packets in the network. The rate is high in the multi-hop routing protocols.

Idle listening mode is one of the significant reasons increasing energy consumption in the network. When node is active but does not receive any packet or sense any event, it wastes the self-energy extremely. This problem is solvable by different methods as sleep/wake up and MAC protocols. The sleep/wake up scheduling is important case in the protocols. For example, in some of the approaches, the nodes are in sleep mode and upon sense or receive data are changed to active position. In another approaches, the modes changing of each node are depend to time. In methods based on MAC, TDMA, contention based and hybrid schemas manage it. In TDMAbased methods, each node has a time slot and uses it to switching modes. Each of the approaches is discussed in the 
following in classification of energy efficiency format. We do not discussed much on the energy-aware data link layer (MAC) protocols. We can say only that MAC protocols are based on content-free and content-based approaches. Content-based approaches have competition for share channel such as MACA and MACAW. In content-free approaches, the channel divides to some sections and each sensor node uses of self-bandwidth without competition. As mentioned in chapter one, some of the content-free approaches are were expressed.

The sensor nodes consume a lot of the energy when they use the control packets. Therefore, the packets number must be managed and the nodes should not use the packets as possible. The packets generally are used in the systems that its goal is reliability. ACK and NACK packets are the samples of the control packets.

According to what was said, energy is one of the most critical resources for WSNs but one problem common to most of them is lack of reliable power for each sensor node in the network. Essentially, data transmission consumes much more energy than data processing. However, the energy consumed by the sensing subsystem varies depending on each node. In some cases, sensing consumes less energy than the one required for data processing while in other cases, it even consumes more than the energy needed for data transmission. In view of the above, several research works has been carried out to solve the energy problem, which results in different schemes and protocols. Most energy conservation techniques in the networking and sensing subsystem is proposing energy efficient protocols to minimize energy consumption during network activities and power management schemes for switching off idle node components are necessary for maximum energy conservation in wireless sensor networks.

We focus on energy efficiency issue in this paper and one of the main our goals is energy saving and prolonging network lifetime. For example, chapter four will propose a new routing algorithm with rely on data-driven and sleep/weak up of nodes techniques. The chapter five will explain a new routing protocol base on machine learning technique that use of datadriven technique of energy efficiency schemas. The chapter six will introduce a novel routing algorithm based on topology control technique. All the techniques are discussed in this chapter completely.

We can classify energy efficient schemes and protocols in WSNs. They are into three classifications so duty-cycling, data-driven and mobility-based methods. Duty cycle schema focuses on subsystem networks and radio transmission switching. Main work of duty-cycle base approaches is maintenance radio transceiver in low power state by sleep mode and it is realizable whenever a sensor node does not communicate with other nodes. If a node is idle and does not senses/sends/receives, the radio mode of the node will wake up to energy consumption management.

Process unit of sensor node do exchanging sleep to wake up mode or vice versa in special and defined periods. This task is done a sleep/wake up scheduling algorithm within any protocol based on duty cycle schema. It is typically a distributed algorithm based on which sensor nodes decide when to transition from active to sleep and back. It allows neighboring nodes to be active at the same time, thus making packet exchange feasible even when nodes operate with a low duty cycle (i.e., they sleep for most of the time). Duty-cycling schemes are typically oblivious to data that are sampled by sensor nodes. On one hand, data-driven approaches are the other method of energy efficiency that can be used to improve the energy saving even more. Data sensing impacts on sensor nodes' energy consumption are in two topics. Sampled data generally has strong spatial and/or temporal correlation [15] so there is no need to communicate the redundant information to the sink. In fact, they are unnecessary samples. The reduction is not enough when the power of self-sensor is low. This issue arises whenever the consumption of the sensing subsystem is not insignificant. Data driven techniques presented in the following are designed to reduce the amount of sampled data by keeping the sensing accuracy within an acceptable level for the application. In case some of the sensor nodes are mobile, mobility can finally be used as a tool for reducing energy consumption (beyond duty cycling and data-driven techniques). In a static sensor network packets coming from sensor nodes follow a multi-hop path towards the sink(s). Thus, a few paths can be more loaded than others can, and nodes closer to the sink have to relay more packets so that they are more subject to premature energy depletion (funneling effect) [16].

If some of the nodes (including, possibly, the sink) are mobile, the traffic flow can be altered if mobile devices are responsible for data collection directly from static nodes. Ordinary nodes wait for the passage of the mobile device and route messages towards it, so that the communications take place in proximity (directly or at most with a limited multi-hop traversal). Consequently, ordinary nodes can save energy because path length, contention and forwarding overheads are reduced as well. In addition, the mobile device can visit the network in order to spread more uniformly the energy consumption due to communications. When the cost of mobilizing sensor nodes is prohibitive, the usual approach is to "attach" sensor nodes to entities that will be roaming in the sensing field anyway, such as buses or animals. The classification is shown in Fig. 3 with detailing of subgroups [17].

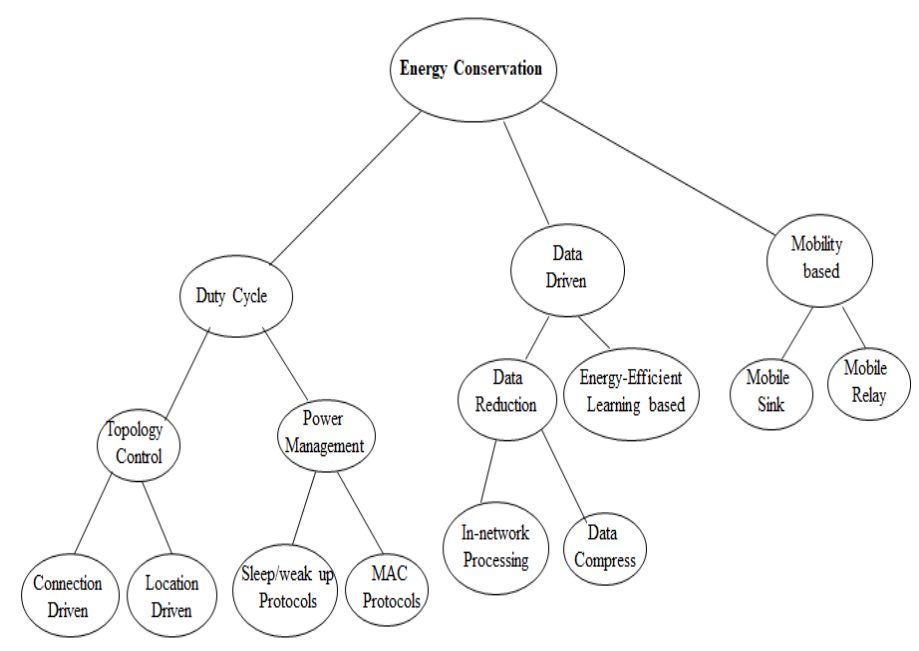

Fig. 3. Classification of energy efficiency schemas in WSNs [7]. 


\section{PROPOSED SYSTEMS}

We propose a new routing algorithm to optimize energy consumption in the WSNs. The protocol is based on a hierarchical approach and it uses a tree structure for deploying network and routing on it. We show that our algorithm has the improvement rather to some of the current methods such as LEACH [15], Improved-LEACH [16], EESR [17] and DHCO [18] in similar fields. Our protocol (EESTDC) has two main phases. The first phase is consisting of steady cluster, Cluster Head $(\mathrm{CH})$ election and Spanning Tree (ST) creation in each cluster and the second phase is data routing. Our protocol is based on a dynamical model in $\mathrm{CH}$ selections, changing topologies structures in any round of running network. This can reduce overhead and improves resources consumption in the whole system.

Structure of communications between sensor nodes in the clusters is based on spanning tree. The tree is structured in the way that the node with the smallest identifier is chosen as the root. All other nodes are connecting to this selected root via the shortest-path route. $\mathrm{CH}$ election is based on specific method that uses the residual energy parameter. In the method, overhead over a $\mathrm{CH}$ node will be reduced because $\mathrm{CH}$ nodes are changed in per round. Routing phase in our algorithm is based on spanning tree algorithm. In fact, it uses a new approach for data transferring from BS/sink to other sensor nodes or vice versa. Tree structure is applied to every cluster after determination $\mathrm{CH}$ nodes. This architecture helped data aggregation in each layer. Therefore, data aggregation task did not impose on the $\mathrm{CH}$ nodes. In addition, it used the TDMA [19] technique in data transferring phase. For example, if a cluster is consisting of 10 sensor nodes (9 nodes and one $\mathrm{CH}$ node) then $\mathrm{CH}$ will cut bandwidth between nine nodes by the TDMA.

$\mathrm{CH}$ election method is shown in the following formula. The first part of this formula is similar to $\mathrm{CH}$ election in the LEACH protocol. Heavy duty imposed on a $\mathrm{CH}$ node is an important weakness in the LEACH and it does not change $\mathrm{CH}$ node in the whole network lifetime. These problems are solved by added parts to formula in this case that is shown in (1).

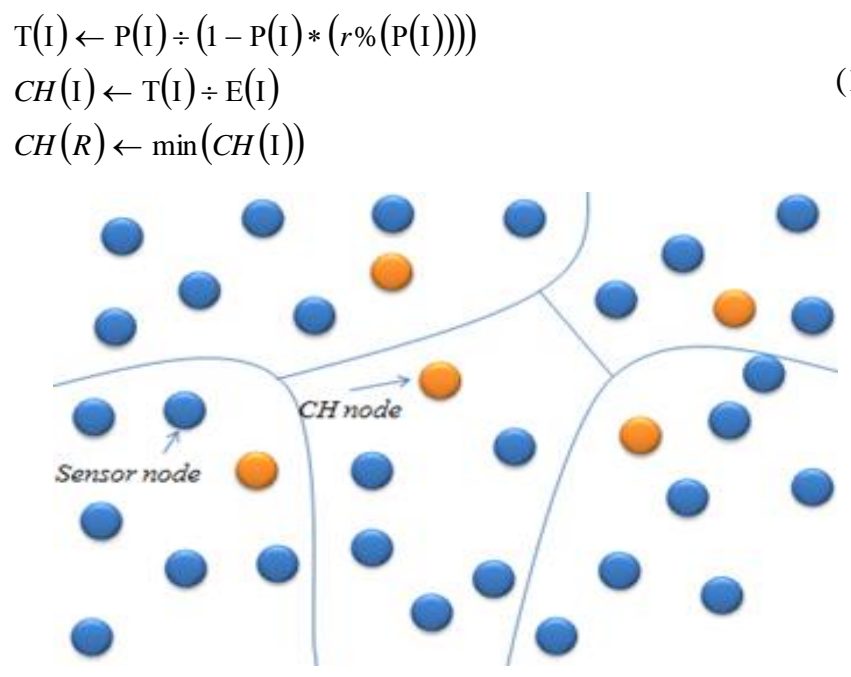

Fig. 4. An example of network after selection CH Nodes.
In this case, $\mathrm{P}$ is a random number that identifies percent of $\mathrm{CH}$ node possibility. $\mathrm{R}$ show current round and $\mathrm{T}$ (I) is a threshold that its value is between zero and one. This value calculates for all sensor nodes in every cluster. I is number of each sensor. E (I) is residual energy of $i^{\text {th }}$ node. In finally, a node is selected as $\mathrm{CH}$ node that has minimum value among $\mathrm{CH}$ (I). This model will cause to reduction energy consumption on $\mathrm{CH}$ nodes, energy balance of the whole network and prolonging lifetime of network. Fig. 4 shows an example of clusters formation and selected $\mathrm{CH}$ node to every cluster.

After election $\mathrm{CH}$ node process, selected $\mathrm{CH}$ node broadcast an announcement message to neighbors nodes that it had selected as $\mathrm{CH}$ node. Selected node is a $\mathrm{CH}$ node for one round only. After receiving message by the each sensor node, it decides be a member of a cluster so it will transmit its data to $\mathrm{CH}$ node of the cluster. If a sensor node has same distance from two or more $\mathrm{CH}$ node then it selects a cluster by random. Tree structure is applied to every cluster after determination $\mathrm{CH}$ nodes. This architecture helps data aggregation in each layer. Therefore, data aggregation task does not impose on the $\mathrm{CH}$ nodes only. This will cause to reduction of system overhead and increasing network lifetime. The architecture has variable topology due to changing in $\mathrm{CH}$ nodes in each round. The nodes must be configured in the range new $\mathrm{CH}$ nodes for reach to acceptable level in the re-organization of tree in the each round. Hence, not all structure of the tree changes in different rounds of running network. The creation of tree is an iterative procedure. Every sensor node is located within a cluster and has a relation with its $\mathrm{CH}$ node. This case is done by tree spanning model. Indeed, this model makes all communications between nodes and nodes- $\mathrm{CH}$ nodes. Each node selects its children and this case is iterative to end. In the end, all sensor nodes are within a tree structure so this structure is based on spanning tree algorithm. After the determination of communication channel of each node, every one of them is active for a few seconds and then is changed to sleep mode. This is a method for energy efficiency in the WSNs so it can avoid unnecessary consumption of energy in each node and this will cause to prolonging lifetime of the network. A sensor node has three main parts as sensing unit (S.U), processing unit (P.U) and transmission unit (T.U) [2]. Generally, T.U and S.U can sleep but P.U is always on in the all conditions. When S.U goes to sleep mode, it buffers sensed data so it can send them after changing mode to wake up. The waking of the S.U is task of the P.U. For example, sensor is activated once every ten seconds by the P.U. In the sleep mode, memory part of P.U may be in sleep mode for more energy saving but it must wakes up after activation of S.U. On the other hand, T.U cannot send or receive any data packet of its neighbors or BS/sink in sleep mode. After activation of node`s RF, it can send the buffered data to its neighbors [8]. The crucial point is that while T.U is sleep mode, node never cannot receive or send data and this case is problematic especially in the target tracking applications. One of the good transmitter modules for short-range schemas is TR1000 module. It has a short range but energy consumption in receiving part is quarter of send part. Indeed, we can hold off the portion of the reception to receive possible message packets of neighbors. Therefore, they can receive wake up or target tracking messages of neighbors. This case is a semi-sleep model and it can active reception part 
of T.U. Our protocol uses the module because it is suitable in target tracking applications such as possible prosecution of enemy tanks. Communications between nodes within each cluster are hierarchical-based and transmissions are from down (child node) to up (parent node). The data packets are aggregate by each the receiver nodes. Hence, $\mathrm{CH}$ node will receive low volume data packet of children and this will cause to prolonging the survival rate of the network. In fact, our protocol increases network lifetime and is energy efficient in three visions. The $\mathrm{CH}$ nodes will be available more time and they will have long-lifetime in the network. This is the result of two reasons. First, reduction tasks over $\mathrm{CH}$ node by data aggregation technique in per nodes. The second is repeated changes in clusters of heads task. Second vision is sleep/wake up approach that uses a specific module for our application types. Third vision is sleep mode in some nodes that they are unrelated to the target. Fig. 5 shows an example of executable schema of EESTDC after finish second phase in a random current time. Fig. 6 represents a pseudo code for set-up and data transferring phases of protocol.

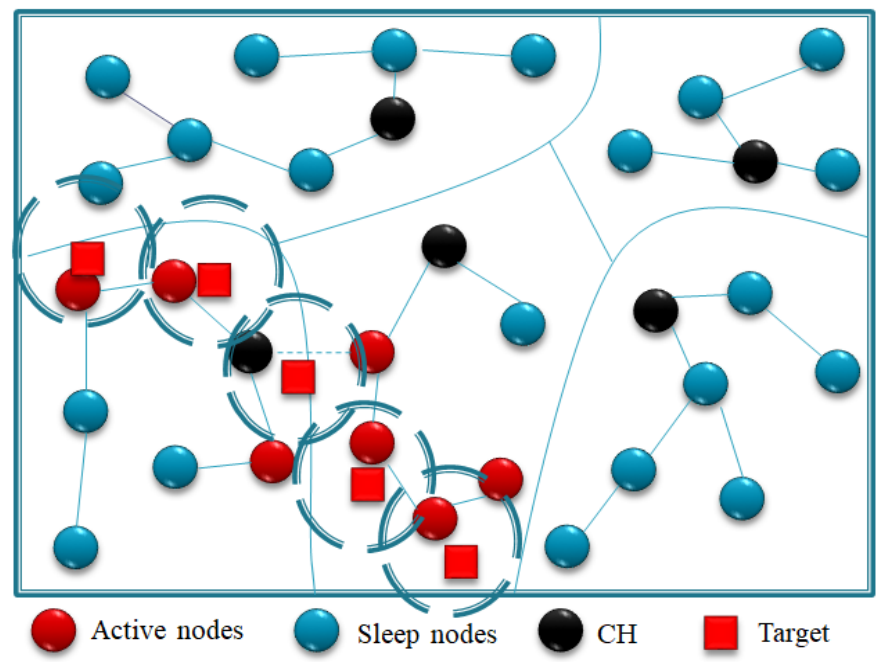

Fig. 5. Example schema from operation in target tracking application after termination phases (CH Node is Active Mode).

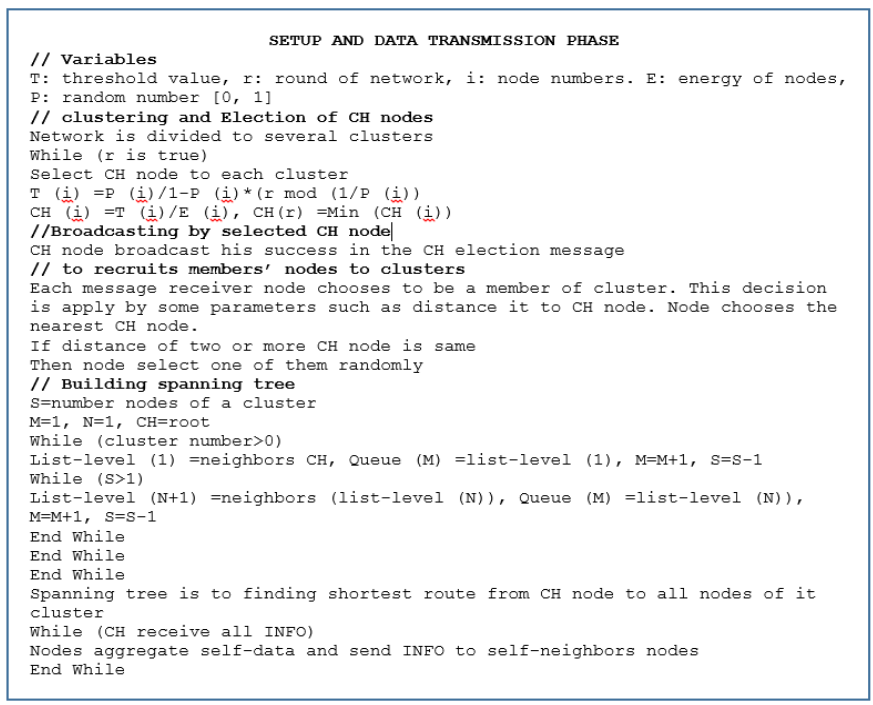

Fig. 6. Overview of pseudo code for setup and data transmission phases.

\section{RESUltS AND EVALUTION}

We made a WSN simulation tool in C\# program that uses it for all simulations in this paper, as shown in Fig. 7. The tool allows us to have results documentation or simulation charts in network lifetime, packet delivery and packet delay parameters at the moment or end. In this case study, we simulate all protocols with the same outputs parameters too such as network lifetime, packet delivery, packet delay and network balance. In addition, we use the original simulation charts of Improved-LEACH, DHCO, and EESR to demonstrate the correctness of the proposed protocol. We apply their input parameters for EESTDC to comparison and then will use our tools for further simulations.

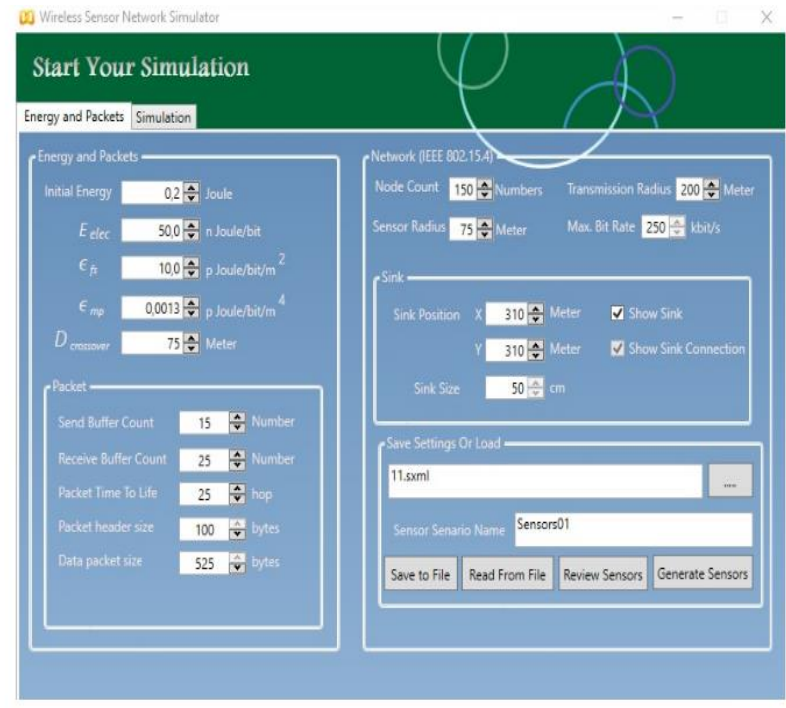

a) Simulator Setting

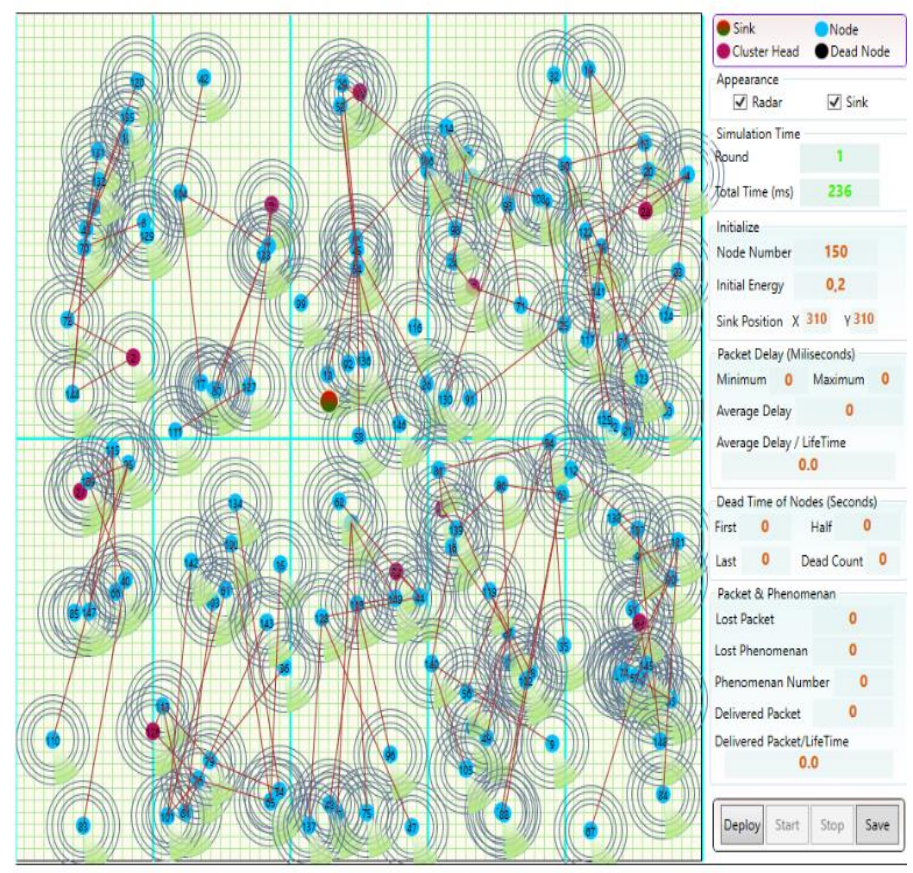

b) Snapshot of simulator program

Fig. 7. A snapshot of basic network simulator. a) Simulator setting, b) Work of the program. 
TABLE I. VALUES OF INPUT PARAMETERS FOR EESTDC, IMPROVEDLEACH, EESR AND DHCO PROTOCOLS

\begin{tabular}{|l|l|l|l|}
\hline $\begin{array}{l}\text { Initial (max) } \\
\text { energy }\end{array}$ & $0.2 \mathrm{~J} / \mathrm{bit}$ & $\begin{array}{l}\text { Receive buffer } \\
\text { size }\end{array}$ & 1500 bytes \\
\hline $\begin{array}{l}\text { Radio/ Sensor } \\
\text { energy } \\
\text { consumption }\end{array}$ & $50 \mathrm{n} \mathrm{J/bit}$ & Send buffer size & 1500 bytes \\
\hline $\mathbf{E}_{\text {elec }}$ & $30 \mathrm{n} \mathrm{J} / \mathrm{bit}$ & $\begin{array}{l}\text { Deployment } \\
\text { area size }\end{array}$ & $(600 \times 600) \mathrm{m}$ \\
\hline $\mathfrak{f}_{\mathrm{fs}}$ & $10 \mathrm{p} \mathrm{J} / \mathrm{bit} / \mathrm{n}^{2}$ & $\begin{array}{l}\text { Send/receive } \\
\text { buffer counts }\end{array}$ & 10 \\
\hline $\mathfrak{E}_{\mathrm{mp}}$ & $\begin{array}{l}0.0013 \mathrm{p} \\
\mathrm{J} / \mathrm{bit} / \mathrm{n}^{4}\end{array}$ & D crossover & $75 \mathrm{~m}$ \\
\hline Data packet size & $150 \mathrm{bytes}$ & Sink position & $(310 \times 310) \mathrm{m}$ \\
\hline Sensing Radius & $4.5 \mathrm{~m}$ & $\begin{array}{l}\text { Transmission } \\
\text { Radius }\end{array}$ & $9 \mathrm{~m}$ \\
\hline
\end{tabular}

The complexity of our work is $\mathrm{O}(\mathrm{cnd})$ that $\mathrm{c}$ is packets amount, $\mathrm{n}$ is number of network nodes and the $\mathrm{d}$ is $\mathrm{d}$ indicates the number of neighbors of each node. We assume the input parameters values have listed in Table I. We ran each of protocols in seven cases with different node numbers. As it seems, EESTDC has a good performance in network lifetime and can increase this factor by methods such as sleep/wake up, data aggregation, applying the new approach in $\mathrm{CH}$ node election and spanning tree methods. Fig. 8 illustrates the results of all four protocols in terms of network lifetime. EESTDC has a good performance than other protocols. The EESTDC improvement is about 6 percent higher than ImprovedLEACH, 21.5 percent higher than EESR and 5.8 percent higher than DHCO.

The second case of comparison is packet delivery. As mentioned, the number of successfully transmitted packets from a node to sink and their reception by the sink is the concept of packet delivery. In addition, packet loss is gained from subtraction of all sensed data packets and number of delivered packets. The proposed protocol has low optimization in packet delivery than Improved-LEACH, DHCO, and EESR unlike its improvement in the network lifetime. The improvement is about 3.5 percent higher than ImprovedLEACH, 7.5 percent higher than EESR and 3 percent higher than DHCO. Fig. 9 shows the packet delivery rate for all protocols.

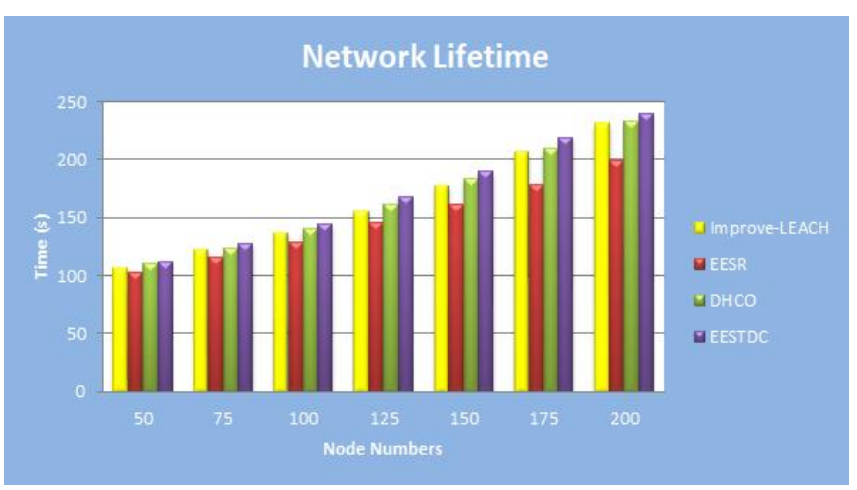

Fig. 8. Network lifetime simulations with different node numbers in improved-LEACH, DHCO, EESR and EESTDC protocols.

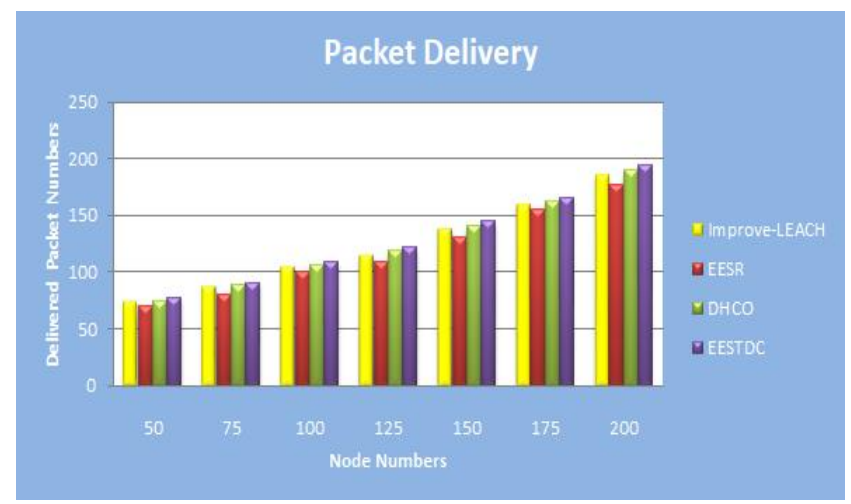

Fig. 9. Network lifetime simulations with different node numbers in improved-LEACH, DHCO, EESR and EESTDC protocols.

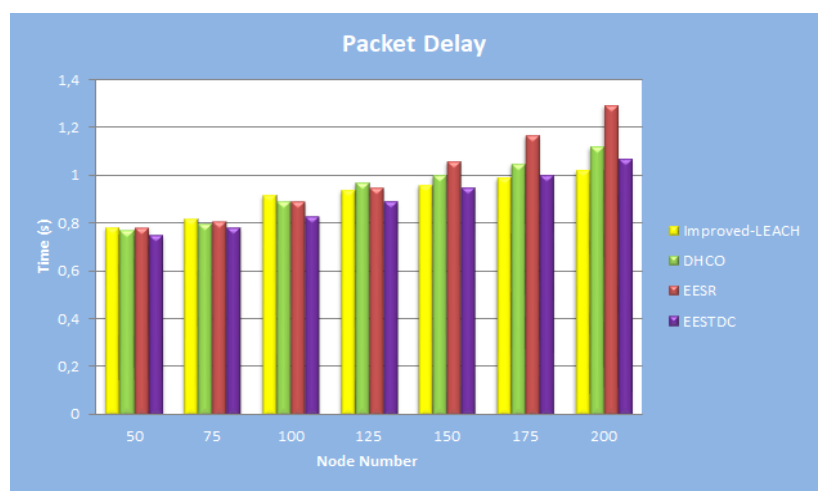

Fig. 10. Packet delay simulations in different node numbers cases for improved-LEACH, DHCO, EESR and EESTDC.

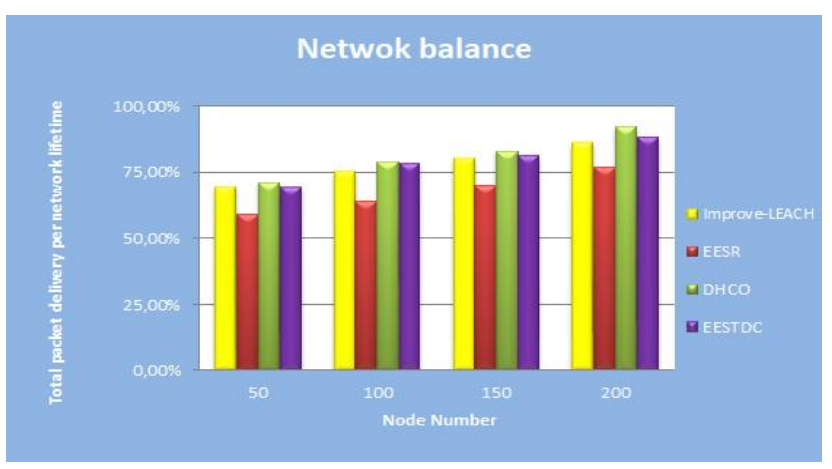

Fig. 11. A specific view from performance of improve-LEACH, DHCO, EESR and EESTDC in network balance.

The third case of comparison is packet delay. As mentioned, packet delay is a period time that a transmitted packet will reach to BS/sink in this time. In fact, a transmitted packet will consume sometimes for reach to BS/sink. This time is a delay for every data packet. Fig. 10 illustrates the simulation results for all fours protocols. As it seems, EESTDC is second suitable approach after Improved-LEACH protocol. Increasing the delay rate in EESTDC has balance.

The last simulation parameter is the network balance. As mentioned, network balance is another measure to the simulation of four protocols. If the main goal of network design is increasing reliability, network lifetime will reduce automatically. With this description, if a protocol can make a balance between output parameters then it would have a good 
performance in general views. It can gain from different methods such as percent of packets delay to network lifetime, percent of packet delivery to network lifetime, etc. Fig. 11 shows a case of network balancing comparison that it is calculated based on the relation between delivered packets numbers and network lifetime. Our expectation from EESTDC is not very good performance because reach to ideal network balance is actually impossible; although, the proposed protocol has an acceptable balance level.

\section{CONCLUSION AND FUTURE WORKS}

As mentioned, energy is an important parameter in the WSNs, hence, the proposed protocol must manage it in the various conditions. Routing algorithms can reduce energy consumption in sensor nodes by finding optimum routes from source nodes to sink and between all nodes. Significant approaches for realizing energy efficiency are hierarchical based routing algorithms that the most applications can use them to reach the goal, however, they have some of the problems such as does not have an optimal network lifetime continually or have overhead over $\mathrm{CH}$ nodes. Each of the methods had some of the advantages and disadvantages, as they were usable in special applications only. Simulation results show the proposed protocol performance in the network lifetime is about 6 per cent higher than Improved-LEACH, 21.5 per cent higher than EESR and 5.8 per cent higher than DHCO. Its improvement in packet delivery parameter is about 3.5 per cent higher than Improved-LEACH, 6.5 per cent higher than EESR and 3 per cent higher than DHCO. In addition, the performance or it in packet delay is about 17 per cent higher than EESR and 6 per cent higher than DHCO but ImprovedLEACH protocol has a good performance than our protocol about 4 per cent.

The proposed protocol can realize energy saving and be prolonging lifetime factors. In addition, EESTDC has suitable performance in the packet delivery parameter. However, levels of network balance and packet delay of EESTDC are medium. The other of the main shortcomings of our protocol is a lack of focus on relationships between the nodes of different clusters. Meanwhile, it seems that $\mathrm{CH}$ node election and restructuring tree in per round of running network has extra overhead over the system. In addition, it seems that an intelligent approach can solve the disadvantages automatically and it tries to keep a balance in the whole of the network.

As future works, we offer some of the suggestions in the fields. So, one of them is focus on the communication between $\mathrm{CH}$ nodes in intra-network and optimize their relations. The second is using the learning based routing techniques and combining with the proposed method. Lastly, use the fuzzy logic methods to create optimal communication paths and activation of nodes can be proposed.

\section{ACKNOWLEDGMENT}

This project has been achieved in Wireless Sensor Networks and IoT Laboratory in Istanbul Sabahattin Zaim University (IZU). The authors declare no conflict of interest.

\section{REFERENCES}

[1] A. Kumar, T. Sato, "Localization in Wireless Sensor Networks: A Survey on Algorithms, Measurement Techniques, Applications and Challenges", Journal Sensor and Actuator Networks, 6, pp. 1-23, 2017.

[2] F. Kiani, E. Amiri, M. Zamani, T. Khodadadi, and A. Abdul Manaf, "Efficient intelligent energy routing protocol in wireless sensor networks", International Journal of Distributed Sensor Networks, 2015, pp.1-13, 2015.

[3] M. Ouadou, O. Zytoune, Y. Hillali, A. Menhaj, D. Aboutajdine, "Energy Efficient Hardware and Improved Cluster-Tree Topology for Lifetime Prolongation in ZigBee Sensor Networks, Journal Sensor and Actuator Networks, 6, pp. 22-37, 2017.

[4] V. Ramasamy, "Mobile Wireless Sensor Networks: An Overview", Open Access Peer-Reviewed Chapter, Books/Wireless Sensor Networks, pp. 3-19, 2017.

[5] I. F. Akyildiz, Y. Sankarasubramaniam, E. Cayirci, "A survey on sensor networks", IEEE Communications Magazine, 40(8), pp.102-111, 2002.

[6] F. Kiyani, H. Tahmasebirad, H. Chalangari, S Yari, "DCSE: A dynamic clustering for saving energy in wireless sensor network", IEEE International Conference on Communication Software and Networks, pp. 13-17, 2010.

[7] G. Anastasi, M. Coti, M. Frrancesco, A. Passarella, "Energy conservation in wireless sensor networks: A survey", Elsevier, Ad Hoc Network, 12, pp. 537-569, 2009.

[8] F. Kiani, A. Aghaeirad, M. K. Sis, A. Kut, , A. Alpkocak, "EEAR: An Energy Effective-Accuracy Routing Algorithm for Wireless Sensor Networks", Life Science Journal, 10(2), pp. 39-46, 2013.

[9] S. LAI, "Duty-cycled wireless sensor networks: Wakeup scheduling, routing, and broadcasting", PhD Thesis, Virginia Polytechnic Institute and State University, 2010.

[10] M. Arifuzzaman, M. Matsumoto, "An Efficient Medium Access Control Protocol with Parallel Transmission for Wireless Sensor Networks", Journal Sensor and Actuator Networks, 1, pp.111-122, 2012.

[11] H. Khanmirza, "Mitigating energy hole problem with power control in heterogeneous sensor networks", IEEE Conference, 2017 Iranian Conference on Electrical Engineering (ICEE), pp. 736-741, 2017.

[12] F. Kiani, "A Survey on Management Frameworks and Open Challenges in IoT", Wireless Communications and Mobile Computing, 2018, pp.133, 2018.

[13] M. C. Vuran, O. B. Akan, I. F. Akyildiz, "Spatio-temporal correlation: Theory and applications for wireless sensor networks", Computer Networks Journal, 45(3), pp. 245-259, 2004.

[14] F. Kiani, Animal behavior management by energy-efficient wireless sensor networks, Elsevier Journal, Computer and Electronic in Agriculture, 151, pp. 478-484, 2018.

[15] W. Heinzelman, A. Chandrakasan, H. Balakrishnan, "Energy-efficient communication protocol for wireless micro sensor networks", International Conference on System Sciences, pp.1-10, 2000.

[16] J. Y. Lee, K. D. Jung, S. J. Moon, "Improvement on LEACH protocol of a wide-area wireless sensor network", Multimedia Tools and Applications, 76, pp. 19843-19860, 2017.

[17] S. Hussain, O. Islam, "An energy efficient spanning tree based multihop routing in wireless sensor networks", International Conference on Wireless Communications and Networking (ACM), pp. 4386-4391, 2007.

[18] Y. Chang, H. Tang, Y. Cheng, Q. Zhao, B. Li, X. Yuan, "Dynamic Hierarchical Energy-Efficient Method Based on Combinatorial Optimization for Wireless Sensor Networks", MPDI Sensors, 17, pp. 1665-1680, 2017.

[19] T. Kaur, D. Kumar, "TDMA-Based MAC Protocols for Wireless Sensor Networks: A Survey and Comparative Analysis", 5th International Conference on Wireless Networks and Embedded Systems (WECON), pp. 1-6, 2016. 